\title{
The Implementation of Information Sharing Mechanism of Multi-agent Synergic Sensing Technology
}

\author{
Yun Zhang ${ }^{*}$, Yang Chen ${ }^{2}$ \\ ${ }^{1}$ School of Computer Science \& Technology, Xi'an University of Science \& Technology, Xi'an 710054 \\ Shaanxi P. R. China. \\ 2 Thought Works Software Technologies (Xi'an) Ltd, Room 06, 6th floor, Building E Huanpu Science and \\ Technology Industrial Park No.211 8th Tiangu Road, Hi-tech Development District Xi'an, 710077 \\ Shanxi P. R. China. \\ * Corresponding author. Tel.: 008613571980258; email: yunzhang710@163.com \\ doi: 10.17706/jsw.11.10.1054-1061
}

\begin{abstract}
The synergic environment sensing based on multi-agent system, which designed the identification process based on combined sensing and was different from the environment perception technology studied mostly by previous research, has been developed. Developed a multi-agent combat environmental information sharing mechanism based on the confrontation of time asynchronous. Proposed multi-agent collaboration of both time synchronization principles and environmental information transfer mode. Finding ways to tackle environmental modeling method used to combat environmental, real-time data exchange between the multi-agent, for intelligent decision-making trend analysis and transfer. The experiments verify the effectiveness of this Mechanism and stressed the importance of intelligent learning.
\end{abstract}

Key words: Multi-agent, synergic environment sensing; information sharing.

\section{Introduction}

One of the important characteristics of the intelligent agent is that it can respond to the change of environment. According to this important characteristic, intelligent agent need to have ability of environmental perception during the confrontation, only be aware of the current situation of our state and behavior of the other state, in order to decide, forecasting and decision-making, so as to counter the other agent [1]. Therefore, in the two sides of the intelligent confrontation, an initial step is to carry out environmental perception. Multi-agent cooperative perception technology is an important technology in this paper, is the core technology of multi-agent "confrontation". In this chapter, based on multi-intelligence theory and environment perception technology, to design identifying the process of environmental perception that based on unite confrontation situation, to study the multi agent information sharing mechanism and the method to estimate the motion state of each other in the confrontation environment. Realize and complete the study of multi-intelligent cooperative environment perception technology under complex confrontation environment.

\section{The Theoretical Study of Multi-Intelligent Agent Environment Perception Technology}

At present, multi-intelligent agent technology is a frontier subject in intelligent decision support 
technology, and it is an important branch of distributed artificial intelligence research[2]. The goal of "multi-intelligent agent" technology is to divide large and complex system into smaller systems, and systems that are able to communicate with each other and coordinate with each other, and are easy to manage[3]. Inside of multi-intelligent agent, be able not only to be simply thinking, but also to communicate and corporate with other intelligent agents with purpose of sharing and using of resource by efficient way.

In this paper, " intelligent-agent" [4] is a kind of computing entity, this intelligent-agent can not only adapt to the environment, but also can feedback and self learning through the environment during decision making of behavior strategy or action.

The structure of agent, which are based on intelligent reasoning and decision making, is as follows: Agent::=<Aid, P, A, R, Dva, Dar, Rule, see, next, estimate, action>

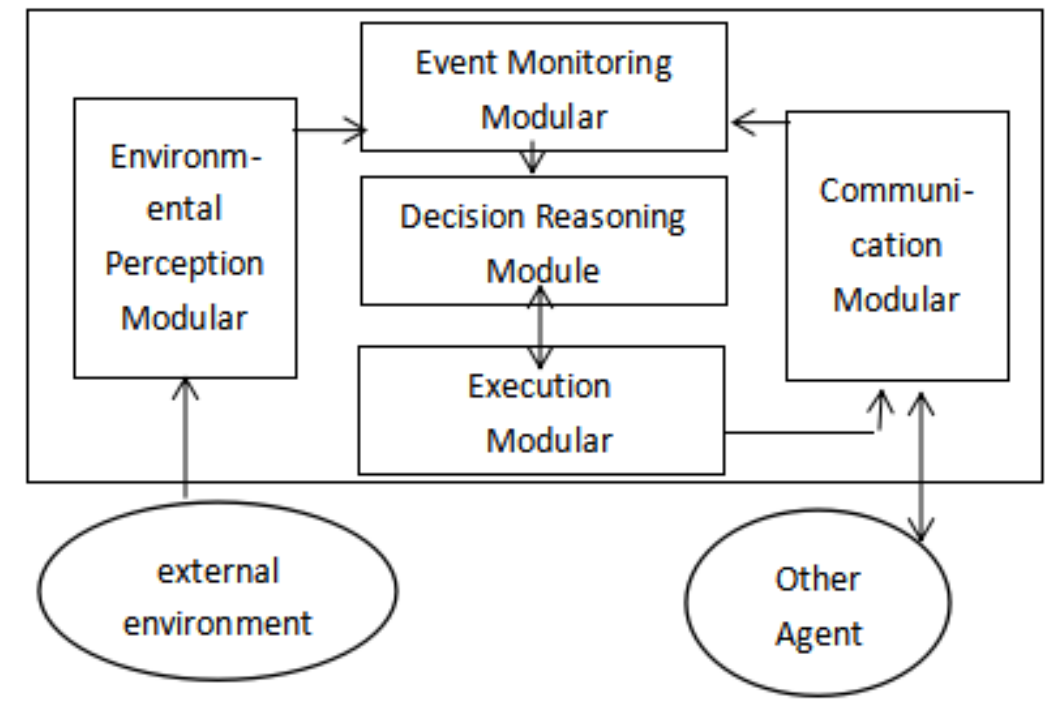

Fig. 1. The basic model of agent.

The meaning of each element of the multivariate group is as follows:

Aid - represents a specific Agent identification;

$P$ - represents the visual state of the Agent set;

A - represents the behavior set of Agent;

See, Next and Action - are used to describe the observation process, thinking process and behavior decision process of Agent;

$\mathrm{R}$ - represents a collection of all possible results states that are likely to be corresponding to the Agent's action plan;

Dva - represents a set of corresponding rules for the visual state and the set of programs;

Dar- represents the correspondence between the scheme set and the result state;

Rule - is a set of specific decision rules;

Estimate - represents a set of selected schemes, which determines the possible result state according to the corresponding rule base of the scheme and the result set;

Execute - represents a set of intent to implement the appropriate behavior[5].

One of the important characteristics of the intelligent agent is that it can respond to the change of environment[6]. Intelligent agent need to have ability of environmental perception during the confrontation. In the condition of knowing the state of current situation and the behavior of each other, intelligent agent would be able to make independent judgment, prediction and decision making and then counter rival with 
solution.

With other auxiliary information, in order to more effectively improve the assessment of the quality, not only to obtain attribute information of target from the environmental monitoring system, but also but also get the target state information (including position, speed, direction of movement, etc.) plus the knowledge of current environmental information, situation assessment. Based on comprehensive utilization the total information, it can improve the accuracy of joint target perception.

\section{Implementation of Multi Agent Information Sharing Mechanism}

Inside multi-agent systems, agents are in an environment that is not able to perform global control or global learning, and these agents may need to cooperate with other agents to achieve their local goals. They can refer to the relevant information of other agents to develop their own behavior strategy, also can send relevant information to other agents to affect the behavior of other agents.

\subsection{Design of Time Synchronization in Multi Agent Counter}

In the process of multi agent game, most of the current environmental information is closely related to the time, the state and behavior of the agent is the time variable. The use of external sensors to capture information, analysis, communication and other needs to spend a certain amount of time, so that the information received from other agents of the environmental information is not the latest, but the history of a moment.

The effect of this time delay is likely to be significant for the intelligence of a highly operational confrontation. For example, with a speed of $2 \mathrm{~m} / \mathrm{s}$ to the movement of the agent, the time delay of 0.1 seconds can be caused by $0.2 \mathrm{~m}$ modeling error. Therefore, to obtain accurate environmental information model, need to consider the information of the time delay factor. So the time must be synchronized in the team [7].

Within intelligent multi-agents counter, need build a commander management system in both sides, the time synchronization between intelligent multi-agent is triggered by commander management system, as shown in Figure 2. The commander management system sends out the time synchronization command, each intelligent agent carried on the time synchronization processing after receive message, and then response message to commander.

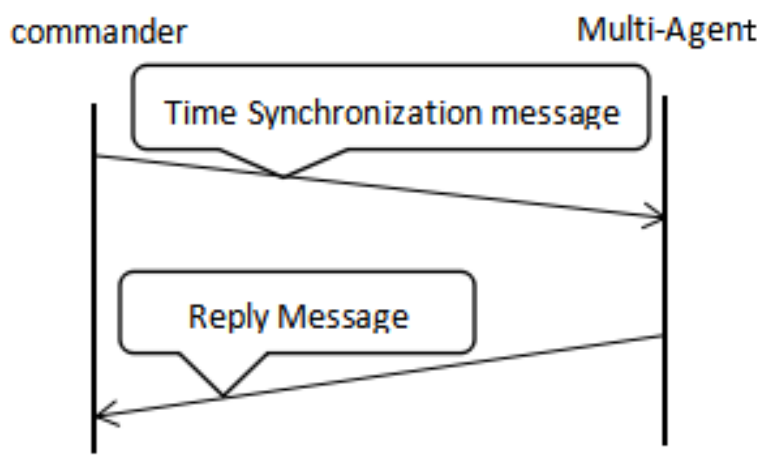

Fig. 2. Time synchronization protocol.

The team time synchronization process is as follows:

1) Commander broadcast time synchronization instructions, including news: the number of synchronization instructions, commander software where the computer system for the high resolution time ta (by the high resolution of the system clock count and system per second include 
high resolution clock counting integrated into).

2) Start timer $T$;

3) The agent after receiving the time synchronization instructions, query their own high resolution time TR, to synchronize

4) The agent sends a time synchronization response message to the commander (the message content is mainly: This response is regarding how many time of synchronization instructions);

5) Tarrived after, commander check whether has the third time synchronization. If not, return to step 1 to continue for a time synchronization; if you have been three time synchronization, commander examination has received within the team all the agents for the third time synchronization instruction response message, if it is, to the monitoring system a synchronous indicating success; otherwise, sending time synchronization failure alarm;

6) Commander figure out which intelligent agent did not finish time synchronization process. Assuming a agent received three synchronization instructions when high resolution time are $\operatorname{Tr} 1 . \operatorname{Tr} 2, \operatorname{Tr} 3$, the high resolution time of commander are, Ta2, Ta3, calculation formula of the intelligent agent for Latest-Team-Time start time T0 are as follows:

$$
t_{0}=\frac{\left(\left(t_{r 1}-t_{a 1}\right)+\left(t_{\gamma 2}-t_{a 2}\right)+\left(t_{r 3}-t_{a 3}\right)\right)}{3}+t_{a 3}
$$

In fact, in most cases, the communication is not reliable, in the calculation formula, the average difference is determined according to how many times the agent receives the synchronization instructions.

\subsection{Implementation of the Method of Transferring Environmental Information}

As mentioned before, when confrontational intelligent agent constructing the global environmental information modeling, not only need their own environmental information, but also need to get the environment information from the allies to carry out modeling. In addition, in order to achieve the tacit cooperation between the agents in the confrontation, it is more important that each agent needs to maintain a consistent understanding of the "environment". This means that the agent needs to know the perception of the environment information of other objects, so as to correct and calibrate the degree to which the environmental information is perceived by itself [7]. Therefore, intelligent agent of the team needs to pass each environmental information.

In the confrontation, the teammate of intelligent agent pass each other their own environment information mainly includes the following contents:

1) Commander management system issue a unified time (usage for time synchronization);

2) Intelligent agents self-learning local environment information;

3) Intelligent agent's movement track and trend, including speed, acceleration and so on;

4) Confrontational area is considered to be the teammate of the various identification information, including the object of the coordinates, the object of the running direction, the size of the object and the movement of trend;

5) Confrontational areas is considered to be the opponent's various identification information, including opponent's global coordinates, running direction, the size of the target and the movement trend, etc.

In addition, when modeling the global environmental information, the following information is required:

1) Establish team unity time of environmental information model;

2) The global positioning information of themselves and their teammates;

3) The movement tendency of the agent itself and the team mate; 
4) The global positioning information of the target object;

5) The movement tendency of the target object, etc...

Multi-agent environment information transmission is through these parameters information transfer to complete[8]. Between each agent through continuous environment information transmission to realize the consistency of "environmental" understanding, with purpose of providing the basic information collection for making situation analysis and judgment.

\subsection{Realization of Environmental Information Modeling Method}

In the confrontational environment, not only to know the information and status of our players, but also to be aware of the situation of each other's intelligent agents, therefore, the intelligent agents need to establish a consistent global environmental information model.

In the confrontational environment, the study of unilateral intelligent agent's environment information modeling is the first step, the basic process as shown in Fig. 3.

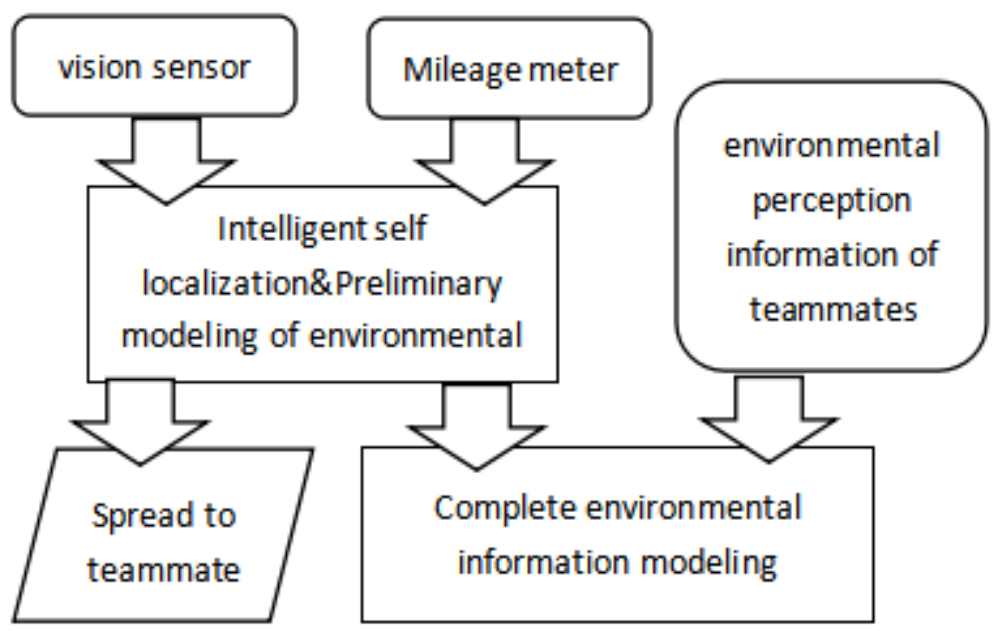

Fig. 3. General schematic diagram of environmental information modeling process.

Step 1: firstly, intelligent agent extracted the the aware data information of image from the "visual" sensor; secondly the intelligent agent fusion extract regional internal fixation maker information and distance sensor information, in order to receive self positioning data, then, obtain the location information of target. Finally, the intelligent agent obtains the self localization information and the preliminary environmental information.

Step 2: intelligent agent (suppose to play as "our" first perspective) based on information fusion with multi-source information, start to track and recognize its sensor data, and construct environment information model.

Step 3: Intelligent agents broadcast the self localization information and preliminary environmental information model in friend agents team range, waiting to receive "teammates" environmental information. (assuming there is not only one agent, but multi-agent cooperation for team contribution, then agents in the same flight is known for his teammates).

Step 4: the intelligent agent fuse its preliminary environmental information model and received his teammates getting environmental information, and comprehensive original historical environmental mode, to establishment of complete the current global environmental information model.

Step 5: To complete the current situation of environmental information perception, all the intelligent agents broadcast complete global environmental information mode within the scope of the players. 
The environmental information collected by a single agent is limited, so it is needed to establish the global environmental information model through the communication and cooperation between the two parties. And obtain accurate, detailed environment information is to make the basis of reasonable decision, therefore, the more accurate the environmental information is likely to make the correct decision [7].

In the process of multi agent confrontation, in order to identify the teammates and opponents, members of the same party intelligent body must be shared by the environmental information to complete the global environment modeling.

The process of global environmental information modeling is shown in Figure 4. Specific processes are described as follows:

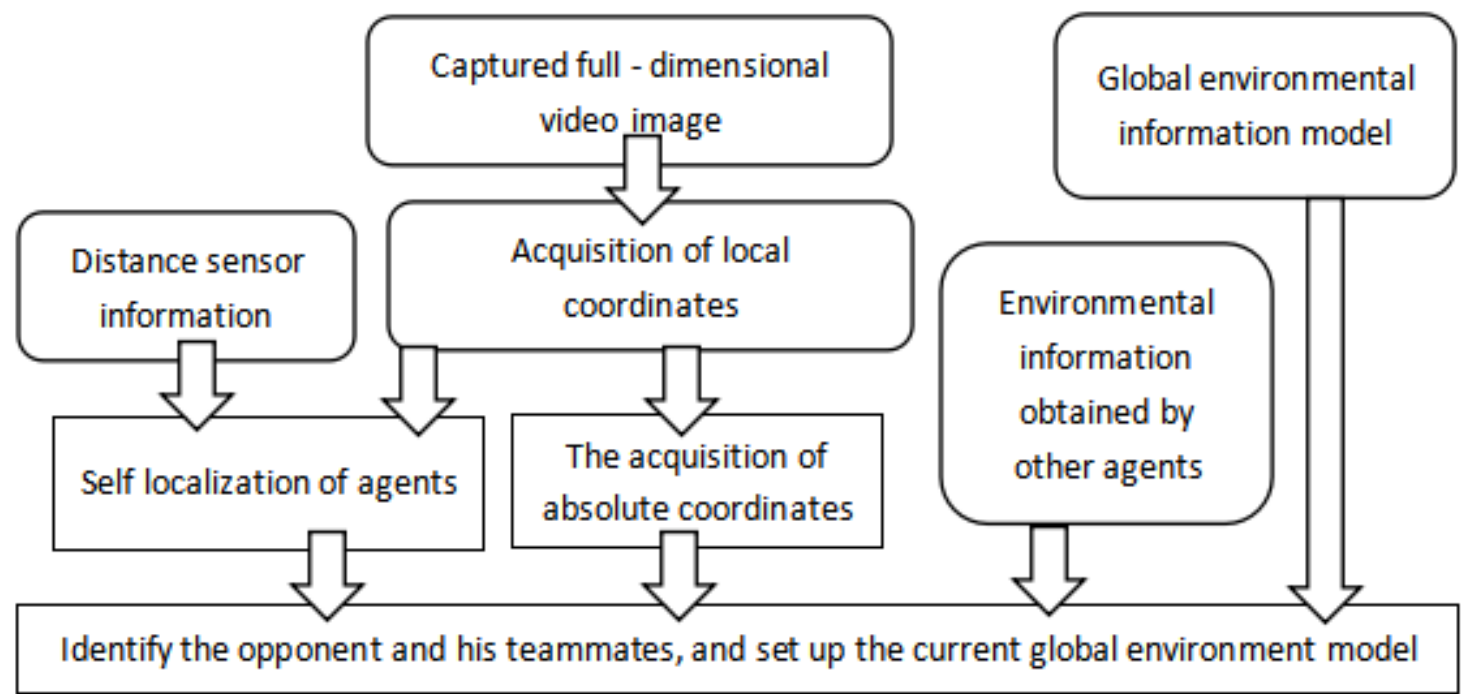

Fig. 4. Framework flow chart of environment information modeling for agent.

1) Intelligent agents capture video images of each dimension;

2) According to the captured video images, isolated from images with various intelligent object corresponding to ID (identification both logos, each agent belongs to the party, each party have unified logo as the difference between the two parties, such as flag or insignia) were obtained the agent's own local coordinate data;

3) The fusion of the data obtained with the distance sensor information, to make the intelligent body of time point of the self positioning information;

4) Self positioning information self checking, filtering to adjust the positioning information, making the self positioning data in the range of error allowed to become more accurate;

5) According to adjust the filter after self localization information, identifies each object of range data by the local relative coordinates into relative battlefield the absolute coordinate system and filter does not belong to the battlefield area within the scope of object identity object;

6) Filtering the information of identified objects

7) To fuse the above information and other intelligent agent's environmental information, and the control of the historical environment information model, construct the current global environmental model.

\section{Experimental Results and Analysis}

As shown in Fig. 5: the system administrator received the three agents of our respective current global environmental information model at a certain moment.

In Fig. 5, our agent 1 (deep red dots in the figure(a)) established global environmental information model, 
in which our four agent coordinates were as follows: $(10.35,5.29,0) ;(9.42,11.38,6.40) ;(14.27,8.34,11.18)$; $(16.94,10.37,6.12)$. The coordinates of the other four agents as follows: $(23.46,8.21,0) ;(24.91,11.46,6.79)$; $(30.09,12.28,5.23) ;(32.33,13.47,4.97)$.

Our agent 2 (deep red dots in the figure(b)) established global environmental information model, in which our four agent coordinates were as follows: $(10.42,5.35,0)$; $(9.71,11.23,6.48)$; $(14.35,8.92,11.30)$; $(16.83,10.49,6.31)$. The coordinates of the other four agents as follows: $(24.28,8.29,0) ;(23.34,11.53,6.87)$; $(30.32,12.35,5.31) ;(32.48,13.30,4.86)$.

Our agent 3 (deep red dots in the figure(c)) established global environmental information model, in which our four agent coordinates were as follows: $(10.64,5.39,0)$; $(9.74,11.31,6.49) ;(14.32,8.83,11.41)$; $(16.79,10.58,6.29)$. The coordinates of the other four agents as follows: $(22.35,7.89,0) ;(23.59,11.62,6.87)$; (30.39,12.98,5.79); $(32.79,13.57,4.59)$.

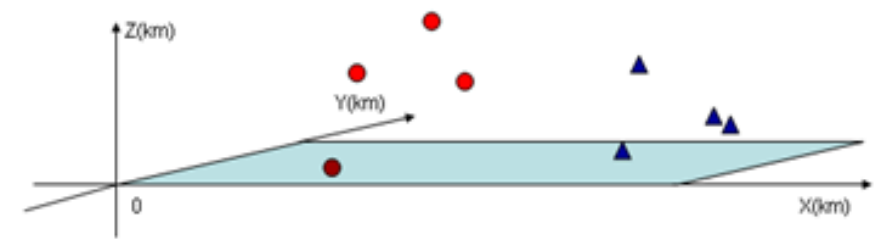

(a)

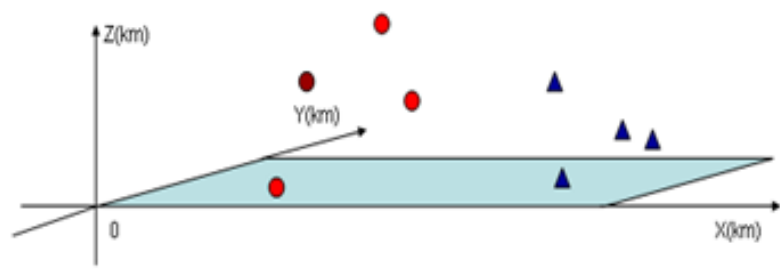

(b)

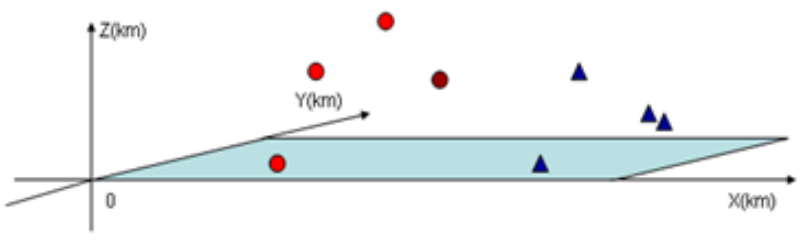

(c)

Fig. 5. The global environmental information model at a certain time which is obtained by our three agents.

However, the actual situation of the control chart display is:The coordinates of our four agents are (10.48,5.36,0), $(9.62,11.34,6.47),(14.35,8.92,11.26),(16.85,10.48,6.26)$. The coordinates of the other four agents as follows: $(23.51,10.29,0),(23.94,11.57,6.87),(30.22,12.68,5.56),(32.51,13.39,4.66)$.

Experiments show that the intelligent body in the recognition of their teammates more accurate, in the identification of the enemy, there is a certain error, the number of errors depends on the visual error and communication delay and other aspects of the factors. In addition, at some point, the agent did not receive the current environmental information processing results, but in the modeling of the early moments of the environmental information processing results, in order to lead to the occurrence of errors.

\section{Conclusion}

In this paper, the research on the multi-agent information sharing mechanism, in multi-agent system, agents in a can't global control or global learning environment, these agents may need and other agents 
work together to achieve its own local goal. In its own development strategy can refer to other agents of the relevant information and other methods, but also to send relevant information and environmental variable to other agents to affect the behavior of other agents of the decision.

In this paper, based on the research of time synchronization and the transfer of environmental information, the modeling method of environmental information is discussed. This is the core of multi agent Environment perception technology. Finally, the experimental results show that the proposed mechanism is effective and inadequate, which provides a theoretical basis and experimental basis for multi agent cooperative perception technology.

\section{References}

[1] Olfatisaber, R. (2015). rm murray, consensus problems in networks of agents with switching topology and time-delays. IEEE Transactions on Automatic Control, 49(9), 1520-1533.

[2] Singh, M. P. (2004). Multi-Agent System: A Theoretical Framework for Intentions, Know-how, and Communications.

[3] Chuang, T. T., \& Yadav, S. B. (2008). The development of an adaptive decision support system. Decision Support Systems, 24(2),73-87.

[4] Wang, Q. W. (2011). Research on Multi Agent Based collaborative Model and Learning Method. Lanzhou Jiaotong University, Master degree thesis.

[5] Xin, H., Qiang, Z., Chen, Z. Z. (2012). Event-triggered average-consensus of multi-agent systems with weighted and direct topology. Journal of Systems Science and Complexity, 25(5).

[6] Yager, R. R. (20070. Intelligent agents for world wide web advertising decisions. Int J of Intelligent Systems, 12(2), 379-390.

[7] Luo, Z. (2007). Key Technologies of Multi-Robot Coordination and Cooperation in Adversarial Environment. Shanghai Jiao Tong University. Doctoral Dissertation.

[8] Shen, Y., Steven, X. D., Adel, H. A. Sari, \& Hao, H. Y. (2013). Data-driven monitoring for stochastic systems and its application on batch process. International Journal of Systems Science, 44(7), 1366-1376

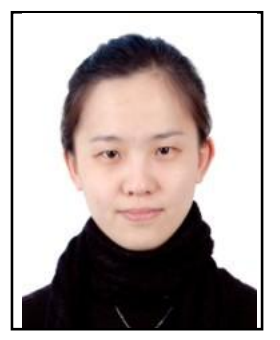

Zhang Yun was born in Xi'an, Shaanxi, China, in 1983. She graduated from School of Computer Science and Technology, Northwestern Polytechnical University (Xi'an Shaanxi, China), She obtained a PhD in computer science and technology in 2014, and the research field is intelligent decision support system.

She worked as a teacher at Xi'an University of Science and Technology after graduation. Work and the research field is intelligent decision support system . 